Disclosure of Interests: Gwenda Simons: None declared, Jorien Veldwijk: None declared, Rachael Di Santostefano Shareholder of: Johnson \& Johnson, Employee of: Janssen R\&D (of Johnson \& Johnson), Matthias Englbrecht Speakers bureau: AbbVie, Chugai, Eli Lilly, Novartis, Roche, Sanofi, Mundipharma, Paid instructor for: AbbVie, Chugai, Roche, Consultant of: AbbVie, Novartis, Roche, Sanofi, Grant/research support from: Roche, Chugai, Christine Radawski Shareholder of: Eli Lilly \& Company, Employee of: Eli Lilly \& Company, Larissa Valor: None declared, Karim Raza Consultant of: Personal fees from Abbvie, Pfizer, Sanofi, Lilly, Bristol Myers Squibb, UCB, Janssen, and Roche Chugai, Grant/research support from: Abbvie and Pfizer, M. Falahee: None declared DOI: 10.1136/annrheumdis-2021-eular.2168

\section{Advances in Pediatric Rheumatology}

\begin{tabular}{|l|l|}
\hline OP0161 & PREDICTIVE VALUE OF MUSCULOSKELETAL \\
ULTRASOUND IN PATIENTS WITH JUVENILE \\
IDIOPATHIC ARTHRITIS IN CLINICAL REMISSION
\end{tabular}

M. Mazzoni ${ }^{1}$, S. Merlo ${ }^{1,2}$, C. Morreale ${ }^{1,2}$, A. Pistorio $^{3}$, S. Viola ${ }^{2}$, S. Ancona ${ }^{1,2}$, F. Magnaguagno ${ }^{4}$, A. Consolaro ${ }^{1,2}$, A. Ravelli ${ }^{1,2}$, C. Malattia ${ }^{1,2}{ }^{1}$ Università degli Studi di Genova, Dipartimento di Neuroscienze, Riabilitazione, Oftalmologia, Genetica e Scienze Materno-Infantili, Genoa, Italy; ${ }^{2}$ IRCCS Istituto Giannina Gaslini, Clinica Pediatrica e Reumatologia, Genoa, Italy; ${ }^{3}$ IRCCS Istituto Giannina Gaslini, Epidemiologia e Biostatistica, Genoa, Italy; ${ }^{4}$ RCCS Istituto Giannina Gaslini, UOC Radiologia, Genoa, Italy

Background: The accurate assessment of remission status in JIA patients is of utmost relevance to taper medications and prevent side effects from their longterm administration. In RA patients in clinical remission (CR), musculoskeletal ultrasound (MSUS) allows to detect persistent joint inflammation (subclinical synovitis), which predicts disease flare and structural damage progression. Although subclinical synovitis has been reported in a substantial proportion of JIA patients with inactive disease, its prognostic value is still being defined. Objectives: 1) to investigate the prevalence of MSUS-detected subclinical synovitis in JIA patients in CR; 2) to establish which and how many joints should be scanned to reliably assess remission; 3 ) to evaluate the persistence of subclinical synovitis over the time; 4 ) to investigate whether subclinical synovitis entails a risk of disease flare and whether it should affect the therapeutic strategy.

Methods: 135 consecutive JIA patients who met the Wallace criteria for CR were included in this 3-years prospective study. All patients underwent MSUS assessment of 56 joints at study entry and at 6 months follow-up visit. Joints were scanned for synovial hyperplasia, joint effusion and Power Doppler (PD) signal by two independent ultrasonographers. Patients were followed clinically for 3 years. A flare of synovitis was defined as a recurrence of clinically active arthritis. The association between clinical and MSUS variables with flare, was evaluated by adjusted logistic regression models.

Results: 135 patients ( $78.5 \%$ F; median age 11.3 y; median disease duration 5.7 $\mathrm{y}$; median CR duration $1.4 \mathrm{y}$ ) were included. Fifty-seven/135 (42.2\%) patients had persistent oligoarthiritis; 41/135 (30.4\%) extended oligoarthiritis; 32/135 (23.7\%) polyarthiritis; $5 / 135(3.7 \%)$ systemic arthritis. Seventy-eight/135 (57.7\%) patients were in CR on medication. Subclinical synovitis was detected in 32/135 (23.7\%) patients and in $53 / 7560(0.7 \%)$ joints. Subclinical tenosynovitis was present in $20 / 135(14.8 \%)$ patients. Subclinical synovitis was found more frequently in the ankle and wrist joints. $58.6 \%$ of patients showed persistent subclinical synovitis at 6 month follow up MSUS examination. During the 3-year follow up 45/135 (33.3\%) patients experienced a disease flare (median survival time $2.2 \mathrm{y}$ ). PD positivity in tendons was the stronger independent risk factor of flare on multivariable regression analysis (HR: $4.8 ; \mathrm{P}=0.04)$. Other predictors of flare were the JIA subtype (oligo-extended form: HR: 2.3; $\mathrm{P}=0.031$ ) and the status of $C R$ on medication (HR: $3.7 ; \mathrm{P}=0.002$ ).

Conclusion: our results confirm that MSUS is more sensitive than clinical evaluation in the assessment of persistent synovial inflammation in JIA patients. Subclinical tenosynovitis was the best predictor of disease flare. To date, the role of tenosynovitis in the diagnosis and prognosis of JIA has been poorly investigated. Our results further support the role of MSUS, especially of the wrist and the ankle, in monitoring JIA patients in clinical remission and to predict disease flare. REFERENCES:

[1] De Lucia O, et al. Baseline ultrasound examination as possible predictor of relapse in patients affected by juvenile idiopathic arthritis (JIA). Ann Rheum Dis. 2018 Oct;77(10):1426-1431.

[2] Filippou G, et al. The predictive role of ultrasound-detected tenosynovitis and joint synovitis for flare in patients with rheumatoid arthritis in stable remission. Results of an Italian multicentre study of the Italian Society for Rheumatology Group for Ultrasound: the STARTER study. Ann Rheum Dis 2018;77:1283-9
Disclosure of Interests: None declared

DOI: 10.1136/annrheumdis-2021-eular.3823

\section{OP0162 IDIOPATHIC ARTHRITIS}

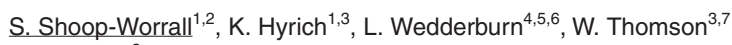

N. Geifman ${ }^{2}$ on behalf of BSPAR-ETN Study, BCRD Study, CAPS, CHARMS,

CLUSTER. ${ }^{1}$ The University of Manchester, Centre for Epidemiology Versus Arthritis, Manchester, United Kingdom; ${ }^{2}$ The University of Manchester, Centre for Health Informatics, Manchester, United Kingdom; ${ }^{3}$ Manchester University NHS FT, Manchester Academic Health Science Centre, NIHR Manchester BRC, Manchester, United Kingdom; ${ }^{4}$ UCL Great Ormond Street Institute of Child Health, Centre for Adolescent Rheumatology Versus Arthritis, London, United Kingdom; ${ }^{5}$ Great Ormond Street Hospital NHS Foundation Trust, Paediatric Rheumatology, London, United Kingdom; ${ }^{6}$ Great Ormond Street Biomedical Research Centre, NIHR, London, United Kingdom; ${ }^{7}$ The University of Manchester, Centre for Genetics and Genomics Versus Arthritis, Manchester, United Kingdom

Background: In children and young people (CYP) with JIA, we have previously identified clusters with different patterns of disease impact following methotrex ate (MTX) initiation. It is unclear whether clusters of treatment response following etanercept (ETN) therapy exist and whether, in a group of CYP who have responded inadequately to or had adverse events on methotrexate, similar treatment response patterns exist. Novel response patterns would aid stratified treatment approaches through better understanding and potential forecasting of more specific response patterns across multiple domains of disease.

Objectives: To identify and characterise trajectories of juvenile arthritis disease activity score (JADAS) components following ETN initiation for JIA.

Methods: ETN-naïve CYP with non-systemic JIA were selected if enrolled prior to January 2019 in at least one of four CLUSTER consortium studies: BSPARETN, BCRD, CAPS and CHARMS, at point of starting ETN as their first biological therapy. JADAS components (active joint count, physician's global assessment $(0-10 \mathrm{~cm})$, parental global evaluation $(0-10 \mathrm{~cm})$ and standardised ESR $(0-10)$ were collected at ETN initiation and during the following year.

Multivariate group-based trajectory models, that identify clusters of CYP with similar patterns of change over time, were used to explore ETN response clusters across the different JADAS components. Censored-normal (global scores, ESR) and zero-inflated Poisson (active joint count) models were used, adjusting for year of ETN initiation. Optimal models were selected based on a combination of model fit (BIC), parsimony, and clinical plausibility.

Results: Of the 1003 CYP included, the majority were female $(70 \%)$ and of white ethnicity $(90 \%)$, with rheumatoid factor-negative JIA the most common disease category $(39 \%)$.

The optimal model identified five trajectory clusters of disease activity following initiation of ETN (Figure 1). Clusters following ETN were similar and covered similar proportions of CYP to those previously identified following MTX: Fast (Group 1: $13 \%$ ) and Slow (Group 2: 10\%) response, active joint count improves but either physician (Group 3: 6\%) or parent global scores (Group 4: 34\%) remain persistently raised and a group with persistent raised scores across all JADAS com ponents (Group 5: 36\%). Compared to the persistent disease cluster, those with greater improvement had lower age and higher functional ability at ETN initiation and those with persistent raised parent global scores had lower ESR levels and were less likely to be RF-positive at ETN initiation.
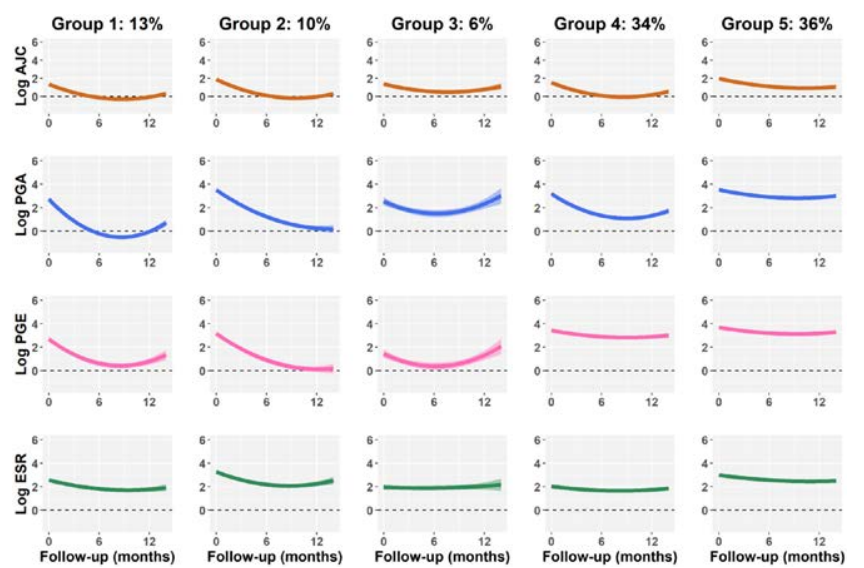

Figure 1. Clusters identified following ETN initiation in children and young people recruited to the UK BSPAR-ETN, BCRD, CAPS and CHARMS studies. 
Conclusion: This study has identified that within CYP initiating ETN, similar response clusters are evident to those previously identified following MTX. This commonality suggests a new framework for understanding treatment response, beyond a simple responder/non-responder analysis at a set point, which applies across multiple drugs despite different mechanisms of action and previous unfavourable treatment outcomes. Understanding both clinical factors associated with, and biological mechanisms underpinning, these clusters would aid stratified medicine in JIA.

Acknowledgements: We thank the children, young people and families involved in CLUSTER, as well as clinical staff, administrators and data management teams. Funding for CLUSTER has been provided by generous grants from the MRC, Versus Arthritis, GOSH children's charity, Olivia's vision and the NIHR Manchester and GOSH BRC schemes.

Disclosure of Interests: Stephanie Shoop-Worrall: None declared, Kimme Hyrich Speakers bureau: Abbvie, Grant/research support from: BMS, UCB, Pfizer, Lucy Wedderburn Speakers bureau: Pfizer, Grant/research support from: Abbvie, Sobi, Wendy Thomson Grant/research support from: Abbvie, Sobi, Nophar Geifman Grant/research support from: Abbvie, Sob DOI: 10.1136/annrheumdis-2021-eular.2505

\section{OP0163 COMPARATIVE ANALYSIS OF ETANERCEPT BIOSIMILAR AND ORIGINATOR USE IN CLINICAL PRACTICE: DATA FROM THE GERMAN BIKER-REGISTRY}

G. Horneff ${ }^{1}$, D. Windschall ${ }^{2}$, T. Hospach $^{3}$, S. Mrusek ${ }^{4}$, M. Rühlmann ${ }^{5}$, A. Klein ${ }^{1}$ on behalf of BIKER-Registry collaborative group. ${ }^{1}$ Sankt Augustin, Paediatrics, Sankt Augustin, Germany; ${ }^{2}$ Sendenhorst, Paediatric Rheumatology, Sendenhorst, Germany; ${ }^{3}$ Klinikum Stuttgart - Olga Hospital / Women's Hospital, Paediatrics, Stuttgart, Germany; ${ }^{4}$ Baden-Baden, Paediatric rheumatology, Baden-Baden, Germany; ${ }^{5}$ Göttingen, Paediatrics, Göttingen, Germany

Background: In 2017, 2 Etanercept biosimilars became approved. Comparative studies performed in adult patients with rheumatoid arthritis, ankylosing spondylitis or psoriasis by extrapolation led to approval for juvenile idiopathic arthritis (JIA)

Objectives: So far there is limited experience with Etanercept biosimilars in JIA: The large national data base of the BIKER-registry was used to describe experience with Etanercept biosimilars in clinical practice.

Methods: In this retrospective analysis patients exposed to ETA were identified in the German BIKER-registry and grouped into cohorts according to initiation of treatment after 2017, use of the originator and of biosimilars. The course of JADAS10, Physician global assessment VAS 0-100-mm, Parent/patient global assessment VAS 0-100-cm, Active joint count 0-71, truncated at 10, ESR and CHAQ-DI was analyzed. Descriptive statistics was used for demographic, clinical data, drug exposure, adverse events (AEs) and events of special interest (ESI).

Results: Until 31.10.2020, 2917 JIA patients were reported to have received Etanercept. Since January 12017 , in 39 centres treatment with Etanercept was started in 439 patients $(377(85.9 \%)$ started with the originator and $62(14.1 \%)$ started a Biosimilar). Biosimilars were prescribed n 17 centres (44\%). In 12 centres $(31 \%)$, Etanercept biosimilars were used first line in 62 patients. In 17 centres (44\%), 63 patients switched for the originator to a biosimilar. 3 patients reswitched from the biosimilar to the originator. 4 patient switched from a biosimilar to the originator). 22 centres (56\%) had not prescribed a biosimilars so far. In not a single centre, initiation of a biosimilar was more frequent than of the originator.

The patients' characteristics and disease activity parameters were widely comparanble. Patients receiving biosimilar first line were slightly older at disease onset and had a longer disease duration. Patients receiving biosimilar first line had more often rheumatoid factor (RF) negative polyarthritis while extended oligoarthritis was more frequent in the originator cohort. In the switching cohort, more patients had extended oligoarthritis and fewer had RF negative polyarthritis and ERA JIA.

No difference in disease activity parameters was noted, neither at baseline, during the course of treatment nor at last observation upon treatment. A decrease of the JADAS10 indicates improvement in both groups (Figure 1). At the time of switching, $68 \%$ had JADAS minimal disease activity (MDA) and $43 \%$ were in JASDAS remission. At month 6 and 12 these numbers increased to $74 \% / 65 \%$ and $62 \% / 50 \%$.

In total, 66 adverse events $(\mathrm{AE})$ were reported in 45 patients upon biosimilar treatment.

33 patients had 1,5 patients 2, 5 patients had 3 and 2 reported 4 events. Adverse event of special interest were hypersensitivity $n=1$, injection site reaction $n=1$, new onset of psoriasis $n=1$, celiac disease $n=1$, Crohn's diesease $n=1$, elevated transaminase $n=2$, depression $n=1$ and disease deterioration (arthritis flare) in $\mathrm{n}=21$. In 20 patients, the etanercept biosimilar was discontinued.
Conclusion: This analysis is the first attempt to present a large data sample on JIA patients exposed to Etanercept biosimilars. Biosimilar were used in a minority of patients and by a minority of centers although no difference in efficacy or safety was noted from our analysis. Until today, the use of the originator is by far exceeding the use of biosimilars. The prescription of a biosimilar either firs line or by switching from the originator is limited to a part of centres. Differences in efficacy between first line biosimilar users and originator users could not be observed. Also, after switching, no loss of efficacy was observed.

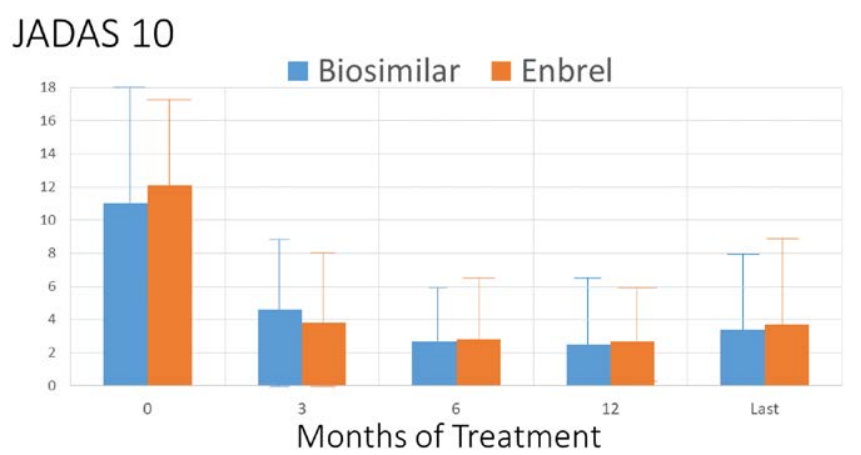

Disclosure of Interests: Gerd Horneff Speakers bureau: Pfizer, Daniel Windschall: None declared, Toni Hospach: None declared, Sonja Mrusek: None declared, Michael Rühlmann: None declared, Ariane Klein: None declared DOI: 10.1136/annrheumdis-2021-eular.1003

OP0164

LONG-TERM SAFETY OF ANAKINRA IN PATIENTS WITH SYSTEMIC JUVENILE IDIOPATHIC ARTHRITIS FROM THE PHARMACHILD REGISTRY

G. Giancane $^{1,2}$, R. Papa ${ }^{1,2}$, S. Vastert ${ }^{3}$, F. Bagnasco ${ }^{4}$, J. F. Swart ${ }^{3}$, P. Quartier ${ }^{5}$, M. Hofer ${ }^{6,7}$, J. Anton ${ }^{8}$, S. Kamphuis ${ }^{9}$, H. Sanner ${ }^{10}$, M. Glerup ${ }^{11}$, F. De Benedetti $^{12}, \mathrm{E}$. Tsitsami ${ }^{13}$, A. Remesal ${ }^{14}, \mathrm{E}$. Moreno Ruzafa ${ }^{15}, \mathrm{~J}$. De Inocencio ${ }^{16}$ C. Myrup ${ }^{17}$, C. Pallotti ${ }^{1}$, I. Koné-Paut ${ }^{18}$, K. Franck-Larsson ${ }^{19}$, H. Malmstrom ${ }^{19}$, S. Cederholm ${ }^{19}$, A. Pistorio ${ }^{4}$, N. Wulffraat ${ }^{3}$, N. Ruperto ${ }^{1} .{ }^{1}$ IRCCS Istituto Giannina Gaslini, Clinica Pediatrica e Reumatologia, Genoa, Italy; ${ }^{2}$ Università degli Studi di Genova, Dipartimento di Neuroscienze, Riabilitazione, Oftalmologia, Genetica e Scienze Materno-Infantili (DiNOGMI), Genoa, Italy; ${ }^{3}$ Wilhelmina Kinderziekenhuis, Department of Pediatric Immunology and Rheumatology, Utrecht, Netherlands; ${ }^{4}$ IRCCS Istituto Giannina Gaslini, Servizio di Epidemiologia e Biostatistica, Genoa, Italy; ${ }^{5}$ Université de Paris, Institut IMAGINE, Centre de référence national pour les Rhumatismes inflammatoires et les maladies Auto-Immunes Systémiques rares de l'Enfant (RAISE), Unité d'Immunologie, Hématologie et Rhumatologie Pédiatrique, Hôpital NeckerEnfants Malades, Assistance Publique Hôpitaux de Paris, Paris, France; ${ }^{6}$ Unité Romande d'Immuno-Rhumatologie Pediatrique, CHUV, University of Lausanne, Lausanne, Switzerland; ${ }^{7}$ Unité Romande d'Immuno-Rhumatologie Pediatrique, University Hospital of Geneva, Geneva, Switzerland; ${ }^{8}$ Hospital Sant Joan de Déu, Universitat de Barcelona, Division of Pediatric Rheumatology, Esplugues de Llobregat (Barcelona), Spain; ${ }^{9}$ Sophia Children's Hospital, Department of Paediatric Rheumatology/Department of Rheumatology, Erasmus University Medical Centre, Rotterdam, Netherlands; ${ }^{10}$ Oslo University Hospital, Dept. of Rheumatology - Norwegian National Advisory Unit on Rheumatic Diseases in Children and Adolescents, Oslo, Norway; ${ }^{11}$ Aarhus University Hospital, Pediatric Rheumatology Unit, Aarhus, Denmark; ${ }^{12}$ IRCCS Ospedale Pediatrico Bambino Gesù, Division of Rheumatology, Rome, Italy; ${ }^{13}$ Aghia Sophia Childrens Hospital, First Department of Pediatrics, University of Athens Medical School, Athens, Greece; ${ }^{14}$ University Hospital La Paz, Rheumatology Unit, Madrid, Spain; ${ }^{15}$ University Hospital Valle de Hebron, Rheumatology Unit, Barcelona, Spain; ${ }^{16} 12$ de Octubre University Hospital, Department of Pediatric Rheumatology, Madrid, Spain; ${ }^{17}$ Rigshospitalet, Pediatric Rheumatology Unit, Copenhagen, Denmark; ${ }^{18}$ National Referral Centre of Auto-Inflammatory Diseases and inflammatory amyloidosis, CEREMAIA, CHU de Bicetre, APHP, University of Paris Sud Saclay, Department of Pediatric Rheumatology, le Kremlin Bicêtre, Paris, France; ${ }^{19} \mathrm{SOBI}$, Swedish, Stockholm, Sweden

Background: Systemic juvenile idiopathic arthritis (SJIA) is characterized by extra-articular manifestations, as fever and rash, and rarely associated by a potentially lethal complication as macrophage activation syndrome (MAS). Anakinra is a recombinant human interleukin (IL)-1 receptor antagonist whose efficacy and safety profile has been studied for patients with SJIA.

Objectives: To evaluate the long-term safety profile of anakinra in patients with SJIA

Methods: Data from patients with SJIA enrolled in the Pharmachild registry before 30 September 2018 and treated with anakinra were analyzed. The study 